\title{
Opportunities and Challenges of implementation of Peer to Peer Block Chain Technology in the Higher Educational Institutions
}

\author{
Afendi Abdi ${ }^{1}$, Dr. Gavendra Singh ${ }^{2}$ \\ HOD $^{1}$, Assistant Professor ${ }^{2}$, \\ Department of Software Engineering, College of Computing \& Informatics \\ Haramaya University, P.O. Box 138 Dire Dawa, Ethiopia
}

\begin{abstract}
According to Merriam-Webster Block chain technology is "an open, distributed ledger that can record transactions between two parties efficiently and in a verifiable and permanent way."

The term "distributed ledger" refers to the fact that the ledger on which transactions are recorded is shared by several participants in the Block chain. As it is not owned or fully controlled by a single entity. Block chain is described as "open", it is typically in reference to the open-source nature of the code base that underlies most Block chain protocols. There are two types of chain exist, public and private chains that are built using open source code

Now a day's Block chain technology is gaining popularity due to its extraordinary features. Block chain is having lots of application in the various fields such as banking, healthcare, Enterprises, agriculture etc. In our research paper we are discussing about the implantation of block chain technology in the higher educational fields and its opportunities and challenges.
\end{abstract}

Keywords: Block chain, private key, cryptography, HEI Here we are using the words like higher educational institutions and Universities interchangeably

\section{INTRODUCTION}

In this technical age, Block chain technology is drawing lot of attention from the research arena and academicians of the various universities worldwide. Block chain technology is a distributed ledger technology (DLT), or we can say that it's an open source distributed database, which is having the characteristics of immutable, transparent and integrity. Block chain technology concept was given by Satoshi Nakamato in 2008, pseudonymous person for removing the third party involvement in the transaction of currency [1].

It is a decentralization technique in which data is not residing in a single server system.it is distributed all over the network. It promotes consensus because it's a record-keeping platform. It's transparent because participants in the chain can download and validate individual ledgers. And it's permanent because those ledgers can't be altered [2].

The Gartner 2019 CIO Survey revealed that $2 \%$ of higher education respondents have already deployed block chain. Another $18 \%$ of respondents were planning to do so within the next 24 months. However, nearly half of respondents (47\%) cited a lack of interest, and that number is up from just $37 \%$ in the 2018 survey [3].

\section{DIFFERENCE BETWEEN CLIENT SERVER AND PEER TO PEER NETWORK}
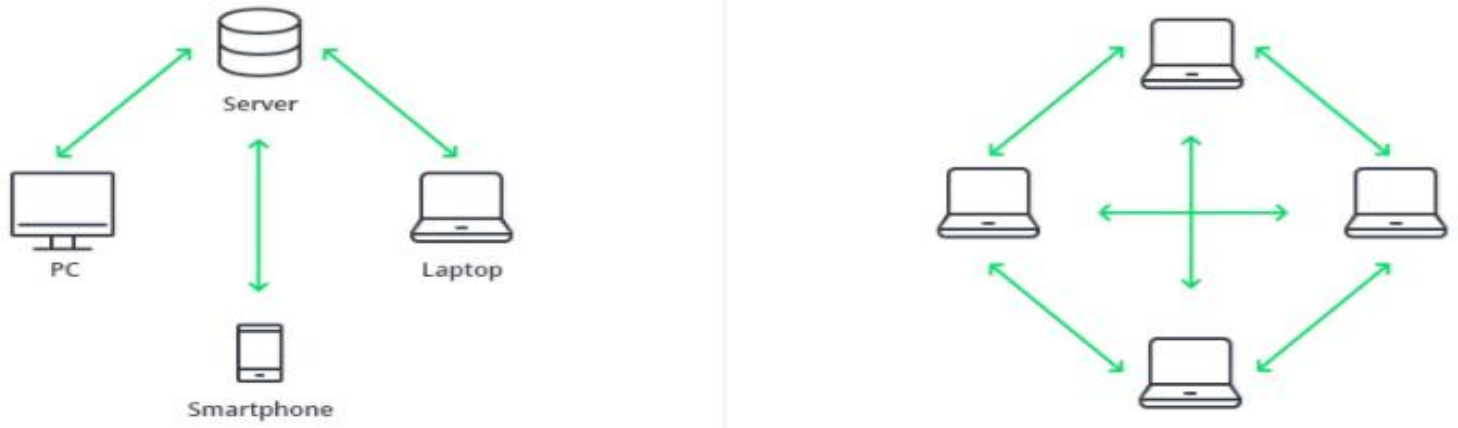

Figure 1: Difference between Client Server and Peer To Peer Network

Resource:https://medium.com/@MLSDevCom/blockchain-architecture-basics-components-structure-benefits-creationbeace $17 c 8 e 77$ 
As the above figure is showing the difference between the difference between traditional database system and this peer to peer network is that in the peer to peer network data is decentralized rather than in traditional database like its residing in the main server

\section{ARCHITETURE OF BLOCK CHAIN WITH DATA STRUCTURE}

The structure of block chain technology is represented by a list of blocks with transactions in a particular order. These lists can be stored as a flat file (txt. format) or in the form of a simple database.

In general, a block chain system consists of a number of nodes, each of which has a local copy of a ledger. In most systems, the nodes belong to different organizations. The nodes communicate with each other in order to gain agreement on the contents of the ledger and do not require a central authority to coordinate and validate transactions

\section{Two vital data structures used in block chain include:}

Pointers - variables that keep information about the location of another variable. Specifically, this is pointing to the position of another variable.

Linked lists - a sequence of blocks where each block has specific data and links to the following block with the help of a pointer [4].

Cryptographic Digital Signature: The public-key cryptography is used in block chain to generate a signature for Block chain transactions. Users carry out transactions by creating a digital signature using their private keys. Recipients in the block chain network verify the transaction using the public key of the sender to ensure that the transaction is indeed signed by the sender.

Distributed Ledger: Block chain use a distributed storage to record the transactions. In essence, all the platforms in the network store either all the transactions or a subset of transactions. All the nodes in the network come to a consensus (using a consensus algorithm) before entering the transactions into the ledger. This feature makes block chain effectively immutable.

Consensus algorithm: Block chain does not rely on a centralized server for verification and validation of transactions. Instead, block chain uses a peer-to-peer model, and all the decisions within the network are made by the participating members through a consensus protocol.[5].

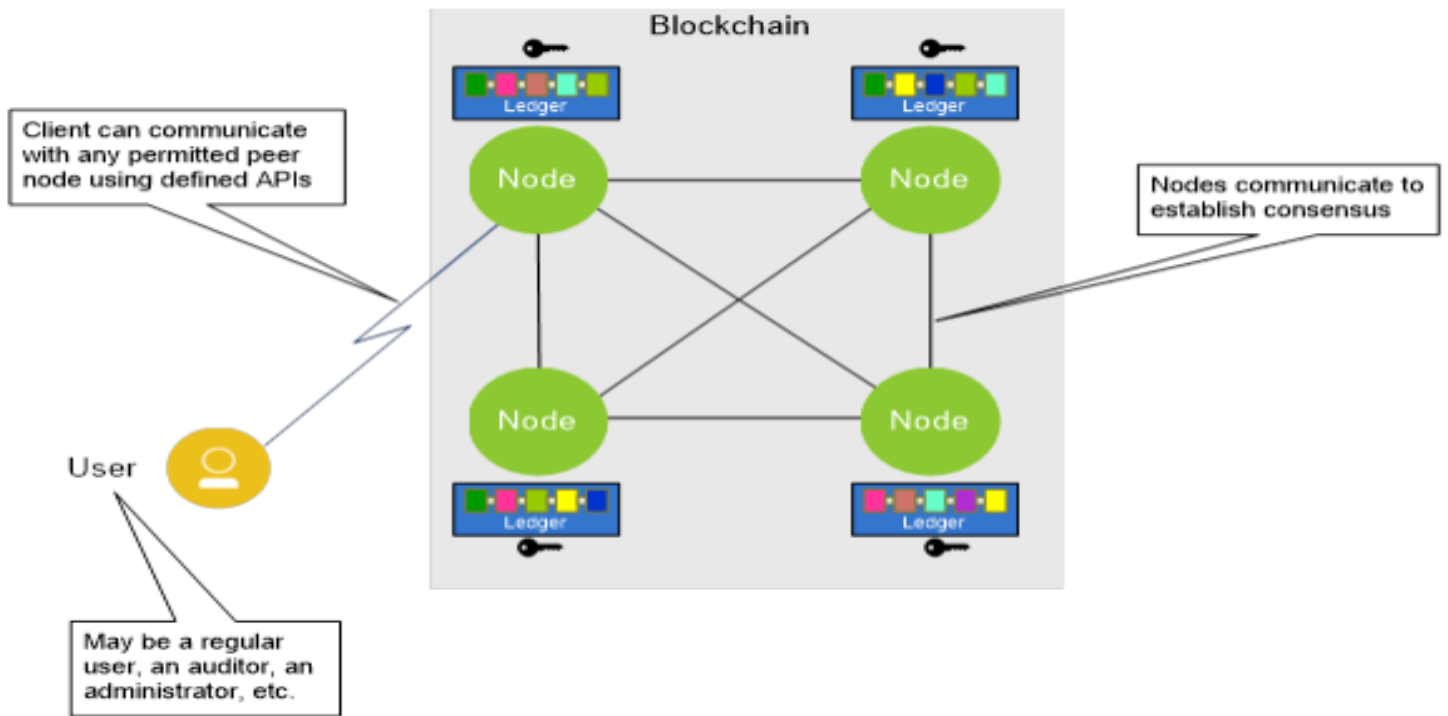

Figure2: Architecture of Block chain

Resource:https://www.omg.org/cloud/deliverables/CSCC-Cloud-Customer-Architecture-for-Blockchain.pdf

\section{RELATIONSHIP BETWEEN BLOCK CHAIN AND CRYPTO CURRENCY}

Mostly people are confused on the terms block chain and crypto currency. For example if we are running Max Video Player application on our Microsoft Windows Operating System. So Block chain is the platform and crypto currency is an application that runs on the block chain platform. Block Chain has a lot of applications in the various fields of HealthCare, Higher Educational Institutions, Banking sector etc.

In our research paper, we will discuss about block chain opportunities and challenges in the field of higher education

\section{BLOCK CHAIN TECHNOLOGY APPLICABILITY ITS OPPORTUNITIES IN THE HIGHER EDUCATIONAL} INSTITUTIONS:

In the growing field of Higher Educational Institution, Block chain is intended to transfer not just content, but also the value inherent in that content. It can bring lot of advantages in the field of higher education. Some universities opting for the block chain technology. The British University in Dubai's (BUiD) Graduating batch of 2017 were the first in the country and third in the world to receive a Digital, block chain Degree during the Graduation Ceremony. These Digital Degrees have already received the proof of Concept (POC) that is the first step in generating a digital certification using the block chain Technology. BUiD will be the first University in the UAE to issue electronic authentication of its Degrees (Masters and Doctoral) via an 
online verification tool. Each certificate fingerprint has also been added to, a decentralized and distributed digital ledger, offering users immediate, secure and digital authentication via the new verification tool on BUiD's website [6].

The University of Bahrain decided to issue and encrypt digital diplomas using the block chain and machine learning technologies in 2019 (University of Bahrain, 2019) [7].

There are so many areas in the field of higher education like academic credentials and online attendance, where we can implement the block chain technology.it will going to be secure and safe and apart from that it's unchangeable

In the Higher Educational Institutions or Universities we can implement the Block Chain technology as there are various fields where we can deploy the Block chain Technology; we are describing some of them as below listed:

\section{(i) Academic Certificates}

When studying at higher educational institutions, student marks are recorded in a document. This is important because academic credentials must be universally verifiable. Verification of the academic credentials from higher educational institutions is largely done manually.

By implementing block chain technology in this area, this process will become online verifiable.so it will be more efficient, as well as militating against duplicitous claims of false credentials universally. Higher Institutions and employers can solve "Fake diplomas and degree" problem and promote Lifelong Learn with verified certificates anywhere in the world on the block chain [8].

\section{(ii) Attendance System}

The Higher educational system can also implement the block chain technology in the attendance management system for both the students as well as employee's also.it will be effective and efficient [9].

\section{(iii) Improving online Course curriculum}

Those are unable to attend the university or higher educational institution classes, taking online courses is a valuable option. Now a day's people are learning the professional courses online. This saves time, money and also commuting.

block chain technology would help decentralise and distribute classrooms globally, enabling a system that exchanges real-time information. This, in turn, would help ensure that the education provided is decentralised, and not solely controlled by an entity who enforces a set syllabus. In effect, a classroom could become a space which is governed by majority consensus [10].

\section{(iv) Transparency in scholarship}

There is a debate within the higher education world centred on who rightfully deserves additional funding in the form of a scholarship. Governments do not have a useful system in place to achieve this. Additional funds are awarded on external factors the government believes are correct. However, situations are circumstantial.

If one student's parents earn a lot of money, that student will receive less funding. If their parents are insistent on not supporting them for whatever reason, that student is at a disadvantage. Another student's parents might earn less money, and cannot afford to support the student. They are also at a disadvantage.

However, a different student's parents might be willing to support them, even on a lower pay check. That student is also likely to receive additional funding because their parents earn a lower amount of money compared to somebody else's parents. This student may then use the additional funding to go on holiday, rather than purchase their textbooks.

Unfortunately, there is not a clear cut, definitive solution to this issue simply because each case is circumstantial. However, block chain technology could help remedy this by ensuring the additional funding is used correctly. If a student spent all of their additional funding without using it to purchase any items that would help them at higher education, it would be apparent on the block chain[11].

\section{(v) Copyright and digital rights protection.}

Block chain's ability to manage, share, and protect digital content makes it ideal for helping researchers, faculty members, and other higher principals create intellectual property, share it, and still control the way it's used. Professors, for instance, could be rewarded based on the actual use, and reuse, of their teaching materials, similar to how they're rewarded based on citations in research papers and journals [12].

Block chain will also be critical in the evolution of "community content repositories" - what we now call libraries. San Jose State University is a leader in the Library 2.0 movement. Block chain could be used in curating digital content and protecting digital rights, among Other Areas [13].

\section{CHALLENGES}

There are so many challenges and problem while we are studying this block chain area. Some of them are as follows:

1. Lack of regulation creates a risky environment

This is a problem with Bit coin or block chain networks. It's a very volatile environment. Due to the lack of regulatory oversight, scams and market manipulation are commonplace.

2. Block chains can be slow:

Once again due to their complexity and their encrypted, distributed nature, block chain transactions can take a while to process, certainly compared to "traditional" or manual systems such as cash or debit cards. For example:Bitcoin transactions can take several hours to finalise, which means there are inherent problems in the idea that you will be able to use them for other works also . And wasn't that something which the "trust less" nature of block chains was expected to remove from the equation?.

3. Consumption of electricity:

It is also one of the major drawback of block chain that's this network consume lot of electricity due to that global warming can also occur [14]. 


\section{CONCLUSION:}

We conclude that the application of block chain technology in the higher educational field is in the very early stage. It's very difficult to say something about it future.howeever it is very much secure ,safe unchangeable and easy for the user but there is no regulation or lack of regulatory makes it little risky for the naïve users. But if implemented with high infrastructure we may have the brightest results in the near future and can solve the main problem of third party involvement in the higher educational field for academics certification verification, offering online course etc.

\section{REFERENCES}

[1] Nakamato S., Bit coin: A peer to peer electronic cash system:2008.//https:bitcoin.org.

[2] https://www.tamimi.com/

[3] https://www.gartner.com/

[4] https://mlsdev.com

[5] Buchman E Tendermint: Byzantine fault tolerance in the age of block chains [Internet]-2016. Available: http://atrium.lib.uoguelph.ca/xmlui/handle/10214/9769, Last Accessed: March 1st, 2019

[6] http://buid.ac.ae

[7] https://www.mei.edu/publications/blockchain-adoption-gulf-states.

[8] https://blockeducate.com/services/blockchain-for-education.

[9] Chintalapati, S., Raghunadh, M.V.: Automated attendance management system based on face recognition algorithms. In: Proceedings of the IEEE International Conference on Computational Intelligence and Computing Research, pp. 1-5. IEEE (2014)

[10] Morrison A. "block chain and smart contract automation: how smart contracts automate digital business, $1: 7$;( 2016$)$.

[11] Xie, H., Wang, J.: Study on block chain technology and its applications. Netinfo Secur. 9, 192-195 (2016)

[12] Sony global education develops technology using block chain for open sharing of academic proficiency and progress records [2016], 2.16. www.sjsu.edu.

[14] www.google.com 